\title{
Unraveling the Influence of Non-Fullerene Acceptor Molecular Packing on Phovoltaic Performance of Organic Solar Cells
}

Yanming Sun ( $\sim$ sunym@buaa.edu.cn )

Beihang University https://orcid.org/0000-0001-7839-3199

Linglong Ye

Xiangtan University

Kangkang Weng

Beihang University

Jinqiu Xu

Shanghai Jiao Tong University

Xiaoyan Du

Friedrich-Alexander-Universität Erlangen-Nürnberg

Sreelakshmi Chandrabose

Victoria University of Wellington

Kai Chen

Victoria University of Wellington

Jiadong Zhou

South China University of Technology

Guangchao Han

Institute of Chemistry

Songting Tan

Xiangtan University

Zengqi Xie

South China University of Technology

Yuanping Yi

Institute of Chemistry Chinese Academy of Sciences https://orcid.org/0000-0002-0052-9364

Ning Li

Friedrich-Alexander University Erlangen-Nü https://orcid.org/0000-0003-1208-4638

Feng Liu

Shanghai Jiao Tong University https://orcid.org/0000-0002-5572-8512

Justin Hodgkiss

Victoria University of Wellington

Christoph Brabec 
Friedrich-Alexander-University https://orcid.org/0000-0002-9440-0253

\section{Article}

Keywords: organic solar cells (OSCs), charge transport

Posted Date: September 21st, 2020

DOl: https://doi.org/10.21203/rs.3.rs-47744/v1

License: (c) (i) This work is licensed under a Creative Commons Attribution 4.0 International License. Read Full License 


\section{Abstract}

In non-fullerene organic solar cells (OSCs), the long-range structure ordering induced by end group $\pi-\pi$ stacking of fused-ring non-fullerene acceptors is considered as the critical factor in realizing efficient charge transport and high power conversion efficiency. Here, we demonstrate that side-chain engineering of non-fullerene acceptors could drive the fused-ring backbone assembly from a $\pi-\pi$ stacking mode to an intermixed packing mode, and to a non-stacking mode to refine its solid-state properties. Different from the above-mentioned understanding, we find that close atom contacts in a non-stacking mode can form efficient charge transport pathway through close side atom interactions. The intermixed solid-state packing motif in active layers could enable OSCs with superior efficiency and reduced non-radiative recombination loss compared with devices based on molecules with the classic end-group $\pi-\pi$ stacking mode. Our observations provide new insights into the influence of non-fullerene acceptor molecular packing on exciton dissociation, charge transport, and recombination losses, and open a new avenue in material design that endows better photovoltaic performance.

\section{Introduction}

Non-fullerene small molecule acceptors (NFAs) that better harness long wavelength radiation and reduce unfavorable interfacial electron coupling lead to fundamental breakthrough in organic solar cells (OSCs) with a remarkable power conversion efficiency (PCE) of over $16 \%^{1-8}$. The well-defined molecular structure and readily controllable variables in chemistry constituted NFAs excellent semiconducting materials by design. The rigid geometry of NFA backbone and flexible side chain result in multiple handles that can be utilized to manipulate NFA solid-state electronic structure to better suit in photovoltaic function, as in results seen from the development of initiative ITIC family to the most recent $Y 6$ analogues ${ }^{9-12}$. The properties of NFAs, including crystallization, molecular ordering and interaction, miscibility, etc. are important parameters that influence the nanostructure and optoelectronic properties of the resulting bulkheterojunction $(\mathrm{BHJ})$ blends ${ }^{13-20}$. $\pi-\pi$ stacking in NFAs is thought as an important factor that controls charge transport, and thus the end group stacking induced long-range order of NFAs is considered as a critical factor governing the merits of organic photovoltaics ${ }^{21-24}$.

Multiple molecular packing motifs are regarded as essential in guiding different transport channels in small molecule organic semiconductors, as seen from previous research of polyaromatic hydrocarbons or thioacences that herring bond structure, one-dimensional slip stack, and two-dimensional brick layer packing induce different transport properties and anisotropy ${ }^{25}$. Such detailed molecular stacking manipulation can hardly be achieved in NFAs, partially due to the large size of NFA molecules that retard molecular crystallization, resulting in technical difficulties to obtain single crystals. Chemistry finds its way to solve these difficulties, of maintaining the backbone to secure electronic structure, and changing the aliphatic side chains to handle the details of molecular crystallization and interaction. This capability enables us to look into the details on how solid-state packing of NFA molecules that affects the electronic structure and function, and to depict a rational structure-property relationship. Such an effort is well 
illustrated in this manuscript, where we designed and synthesized three fused-ring NFAs with the same molecular skeleton, but exhibiting different stacking properties by modifying side-chains, i.e. IDTT-C6-TIC, IDTT-C8-TIC and IDTT-C10-TIC (named IDTT-CX-TIC) (Fig. 1). This material family affords the capability to investigate molecular packing behavior with systematic chemical structure modification. The change in side chain length induces fine-control of aliphatic chain interaction, even in the complicated BHJ blends, which opens the broader frame for backbone $p-p$ reorganization, and thus leads to different solid-state properties. By rationally modifying the length of the side chains, the NFA backbone can be manipulated from a strong p-p stacking mode to an intermixed packing mode, and to a non-stacking mode. Different from our current understanding, we find that close atom contacts in a non-stacking mode can enable efficient charge carrier hopping transport through close side atom interactions. The optimized OSCs free from end group p-p stacking yield a superior PCE of $12.7 \%$ with reduce non-radiative recombination loss to that of OSCs rely on classic end group p-p stacking formed major transport channels, which is not cognized in the organic photovoltaic community. More importantly, molecular and crystal engineering allow to combine the two solid-state packing motifs together in a BHJ blend, leading to a PCE of $13.7 \%$, surpassing the single mode interaction dictated function. We believe these observations are vital in new organic semiconductor material design that better performance can be obtained through detailed crystalline structure manipulation.

\section{Results}

Crystal packing and morphology investigation. The chemical structures of the three NFAs are shown in Fig. 1. The frontier molecular orbital (FMO) energy levels of IDTT-CX-TIC were measured by cyclic voltammetry (CV) and summarized in Supplementary Fig. 1a and Supplementary Table 1. With increasing the length of the alkyl side chains on IDTT-CX-TIC, the highest occupied molecular orbital (HOMO) energy levels gradually decrease (IDTT-C6-TIC: $-5.55 \mathrm{eV}$; IDTT-C8-TIC: $-5.64 \mathrm{eV}$; IDTT-C10-TIC: $-5.71 \mathrm{eV}$ ), and the lowest unoccupied molecular orbital (LUMO) energy levels gradually increase (IDTT-C6-TIC: -3.99 eV; IDTT-C8-TIC: -3.97 eV; IDTT-C10-TIC:-3.91 eV). IDTT-C6-TIC, IDTT-C8-TIC and IDTT-C10-TIC thin films showed similar ultraviolet-visible absorption spectra (Supplementary Fig. 1b) with slightly shifted absorption peak maxima $\left(\lambda_{\max }\right)$ and absorption onsets $\left(\lambda_{\text {onset }}\right)$. Molecule with the medium side chain possesses the lowest optical bandgap $\left(E_{\mathrm{g}}^{\mathrm{opt}}=1.52,1.50\right.$ and $1.56 \mathrm{eV}$ for IDTT-C6-TIC, IDTT-C8-TIC and IDTT-C10-TIC, respectively), caused by their different solid-state molecular packing modes, as illustrated in Fig. 1 (the details of IDTT-CX-TIC single molecular structure are shown in Supplementary Figs. 2-4). In IDTT-C6-TIC, hexyl side chains took a fully extended geometry that stretching out from the conjugated backbone. The adjacent molecules in the $\pi$ plane normal direction are connected through side chain interdigitation, as revealed by the close interaction with an average distance of $10.676 \AA$ in Supplementary Fig. 5. The long axis of the molecules formed a close contact through end-group overlapping, with a distance of $3.546 \AA$ (Supplementary Fig. 6), which falls in $\pi-\pi$ stacking region that facilitates charge transport. The coupling transfer integral was estimated to be $12.7 \mathrm{meV}$ (Supplementary Fig. 7). The overlapping of end group in a long distance $\pi$ plane normal direction yielded a structure feature that one molecule end is inserted in a molecular pair, and the 2D iteration in this manner leads to 
a network that 2D carrier hopping can be achieved. Such a 2D web then overlaid with each other (c-axis direction) to form 3D molecular registration. It is interesting to note that molecular backbones formed slip-stack, that CN group was in quite close distance with adjacent ones ( 3.3 $\AA$, see Supplementary Fig. 5) that weak electronic coupling existed, $9.2 \mathrm{meV}$, marking a 3D transportation scenario in solid state. The simulated carrier mobility of IDTT-C6-TIC is as high as $2.4 \times 10^{-3} \mathrm{~cm}^{2} \mathrm{~V}^{-1} \mathrm{~s}^{-1}$ (see Supplementary Table 2).

IDTT-C8-TIC molecules showed unique solid-state packing as can be tracked from single crystal diffraction. The slightly longer alkyl side chains lead to different molecular geometry in single crystals, as shown in Supplementary Fig. 8, from which we can extract three pairs of interactions, (1) frustrated side chain interaction, (2) symmetric side chain interaction, (3) mixed side chain interaction. In frustrated side chain model, octyl side chains adopted a random extension without structure order, and thus close backbone packing was achieved. In symmetric side chain model, the long contour length would lead to good aliphatic side chain interdigitation as seen in IDTT-C6-TIC, yet a fully extended geometry would result in reduced $p-p$ interaction, such a balance bends over octyl side chain to form a crankshaft structure. The mixed side chain interaction was found between adjacent molecules of the two pairs. Such a complexity leads to an anomalous solid-state packing in single crystal, which could be dissected into two crystalline domains as marked by blue and yellow color region in Fig. 1b. The frustrated interaction contact yielded a transfer integral of $35.2 \mathrm{meV}$ (Supplementary Fig. 9), while the symmetric interaction contact and the mixed interaction model yielded extremely low transfer integral of 0.1 and $0.2 \mathrm{meV}$, respectively. Thus, a highly conductive 1D line was imbedded in a nearly insulating crystalline matrix, making IDTT-C8-TIC unique in NFA materials. There are trivial close atom contacts outside of p-p interaction direction, within partial $0.5 \mathrm{meV}$ range, leading to slow hopping rate for carrier to transport. A low global mobility is expected by integrating all contact types and directions. As a result, the simulated carrier mobility of IDTT-C8-TIC is $2.9 \times 10^{-4} \mathrm{~cm}^{2} \mathrm{~V}^{-1} \mathrm{~s}^{-1}$ (see Supplementary Table 2), almost one order of magnitude lower than that of IDTT-C6-TIC.

IDTT-C10-TIC in single crystal also took two different pairs of interactions, as shown in Supplementary Fig. 10, (1) the harpoon type of side chain extension, (2) the crankshaft type of side chain extension. In both models, a large $\pi-\pi$ stacking distance was recorded with a low transfer integral of close to $0 \mathrm{meV}$ (Supplementary Fig. 11). The molecular packing is similar to the crankshaft-packing motif in IDTT-C8-TIC crystals, and a relatively low mobility is expected since the main carrier hopping channel is through close side atom interactions. The simulated carrier mobility is $6.5 \times 10^{-5} \mathrm{~cm}^{2} \mathrm{~V}^{-1} \mathrm{~s}^{-1}$ (see Supplementary Table 2). Such observation through changing side chain substitution yielded quite interesting structure-property relationship that the molecular signature could be transformed into solid states. IDTT-C8-TIC in crystal is an ordered mixture of IDTT-C6-TIC and IDTT-C10-TIC with a slight difference in stacking slipping. We thus could dictate the solid-state structure of NFAs by this simple but elegant strategy to optimize the optoelectronic properties and performance of organic electronic devices.

It is rational that structure order in thin film can be different from that in single crystal due to the nonequilibrium nature of the casting method ${ }^{20,26-29}$. Polymorph and orientation distribution would add more 
complexity into thin film structure order in grazing incidence wide-angle x-ray diffraction (GIWAXS) results. However, the major molecular packing motif should be translated from single crystal to thin solid films, since the packing mode in single crystal is an energetically favorable state. IDTT-CX-TIC series were quite robust in solid-state packing, and major diffraction peak can be traced from single crystal unit cell with orientation broadening in diffraction peaks (Fig. 2), which were then crosschecked from simulated GIWAXS patterns from specific basal planes. The detailed peak labeling and the related molecular packing motif, for example, the IDTT-C6-TIC (110) crystal plane, the IDTT-C8-TIC (121) crystal plane, and the IDTT-C10-TIC (112) crystal plane, were been presented in Supplementary Fig. 12.

IDTT-C6-TIC presented three diffraction rings at $0.39,0.60,0.72 \AA^{-1}$, corresponding to the (001), $(010) /(100)$ and (1-10) miller planes in single crystal, respectively. The $1.8^{-1}$ diffraction peak corresponded to the p-p stacking, and thus a random orientation was recorded. For IDTT-C8-TIC, more detailed Bragg spots were resolved in small $q$ regions, matching well with the single crystal unit cell. A primary (01-1) peak was seen in in-plane direction, and (121) peak was seen in out-of-plane, indicating a good face-on lamellar order. The (242) plane was seen in $1.60^{-1}$, and it first-order peak (121) was located in lower $q$ region. There were a series of eyebrow peaks that labeled as (011), (021), (120) and (11-2), $(-122)$ miller planes according to single crystal structure and 2D GIWAXS simulated results. A broad diffraction peak was seen at $1.83^{-1}$ in out-of-plane direction, which marked the close p-p stacking in thin film. IDTT-C10-TIC showed a simple diffraction feature with two major rings at 0.80 and $1.60^{-1}$, which corresponded to (112) and (224) miller planes, of the largely spaced p-p stacking distance (7.85). Such a large $\mathrm{p}$-p distance is due to the alkyl chain spacing between adjacent molecules, which is uncorrelated with charge transfer process. Other miller planes were also labeled, which, however, were of much lower intensity, indicating the folded alkyl chain crystallization is the major driving force for such a molecular assembly.

The morphology of PBT1-C:IDTT-CX-TIC blends were studied using the combination of GIWAXS, atomic force microscopy (AFM), transmission electron microscopy (TEM), and resonant soft x-ray scattering (RSoXS) methods. As seen in GIWAXS 2D diffraction patterns and line-cut profiles (Fig. 3), the major structure features in NFAs are preserved in the blends. These samples show typical polymer (100) diffraction at $0.28 \mathrm{~A}^{-1}$. And in IDTT-C6-TIC and IDTT-C8-TIC blends, diffraction peaks at $0.37 \mathrm{~A}^{-1}$ were detected, corresponding to (001) and (011) diffractions in IDTT-C6-TIC and IDTT-C8-TIC molecules. The polymer (100) crystal coherence lengths (CCL) in three samples were estimated to be around $11.5 \mathrm{~nm}$, while the (001) CCL is $15.9 \mathrm{~nm}$ for the IDTT-C6-TIC blend and (01-1) CCL is $11.2 \mathrm{~nm}$ for IDTT-C8-TIC. The IDTT-C10-TIC blend shows quite similar structure as in neat thin film, with a pronounced (112) diffraction at $0.82 \mathrm{~A}^{-1}$, with a CCL of $5.1 \mathrm{~nm}$. The high angle diffraction region is composed of PBT1-C and NFAs p-p stacking information, which cannot be clearly deconvoluted.

Overall, the three blends show well-developed polymer and acceptor crystalline features, which shape the phase separation. The phase images were probed by AFM and TEM (Fig. $3 \mathrm{c}$ and Supplementary Fig. 13). As seen in PBT1-C:IDTT-C6-TIC blends that large-sized aggregations (hundreds of nanometers) 
distributed inside fibril networks. A broad scattering was detected in RSoXS, corresponding to a wide distribution of phase separation lengths with the largest domain size of about $200 \mathrm{~nm}$. These large phases will suffer inefficient charge transfer, leading to suppressed short-circuit current $\left(J_{S C}\right)$ and fill factor (FF) in OSCs. In contrast, the PBT1-C:IDTT-C8-TIC blend exhibited much refined morphology, and a typical fibril network was observed with appropriate phase separation. A scattering hump in RSoXS confirmed the moderate phase separation about $90 \mathrm{~nm}$, suggesting weak aggregation of NFAs in the blends. The combination of fibril network highways and NFA aggregations balance the hole and electron transport in the blend, leading to enhanced charge extraction as well as improved $J_{S C}$ and $\mathrm{FF}^{30-32}$. The PBT1-C:IDTT-C10-TIC blend showed smooth morphology with fibril network and weak phase separation due to good mixing between materials. The electron mobility of $\mathrm{BHJ}$ blend is still as high as $6.7 \times 10^{-5}$ $\mathrm{cm}^{2} \mathrm{~V}^{-1} \mathrm{~s}^{-1}$ (see Table 1), comparable to its neat film (see Supplementary Table 2).

Photovoltaic performance, $V_{\mathrm{OC}}$ loss, and exciton dynamics. To understand the effect of solid state molecular packing on the photovoltaic properties of different BHJ thin films, OSCs based on PBT1C:IDTT-CX-TIC blends were constructed in a device architecture of ITO/ZnO/PBT1-C:IDTT-CX$\mathrm{TIC} / \mathrm{MoO}_{3} / \mathrm{Ag}$. The current density-voltage $(\mathrm{J}-V)$ characteristics of OSCs are displayed in Fig. $4 \mathrm{a}$ and the detailed parameters are listed in Table 1. The IDTT-C8-TIC based devices showed the best PCE of $13.7 \%$ with the highest $J_{S C}$ of $20.3 \mathrm{~mA} \mathrm{~cm}^{-2}$ and the highest FF of 74.6\%. IDTT-C6-TIC and IDTT-C10-TIC based devices exhibited PCEs of $10.0 \%$ and $12.7 \%$ with $J_{S C} S$ of 17.0 and $18.1 \mathrm{~mA} \mathrm{~cm}^{-2}$ and FFs of $66.7 \%$ and $71.3 \%$, respectively. The $J_{S C}$ values estimated from the external quantum efficiency (EQE) spectra (Fig. $4 b)$ are consistent with that obtained from the $J-V$ characteristics. We found that the open-circuit voltage $\left(V_{\mathrm{OC}}\right)$ of OSCs is influenced by the microstructure of BHJ blends, i.e. the molecular ordering of NFAs. Quantification of $V_{\text {OC }}$ losses for OSCs based on the three NFAs is shown in Fig. 4c-e and summarized in Table 2. The bandgaps of OSCs were determined from the intersection of the EQE edge ( $\left(\mathrm{EQE}_{\text {edge }}\right)$ and the local EQE maximum, as shown in Supplementary Fig. $14^{33}$. The bandgap determined in this way takes into account the aggregation of NFAs in the blend films and can avoid underestimation of the bandgap of disordered materials using the absorption onset. IDTT-C10-TIC-blend film has a slightly higher bandgap $(1.61 \mathrm{eV})$ compared to IDTT-C6-TIC $(1.60 \mathrm{eV})$ and IDTT-C8-TIC $(1.59 \mathrm{eV})$, which agrees very well with the absorbance of p-p stacking in the IDTT-C10-TIC blend film. Ideal $V_{\text {OC }}$ estimated according to the ShockleyQueisser limit $\left(\mathrm{V}_{\mathrm{OC}, \mathrm{SQ}}\right)^{34}$ varies within $0.02 \mathrm{eV}$ for OSCs based on the three NFAs. The radiative recombination limit $V_{\mathrm{OC}}, V_{\mathrm{OC}}$, rad, was determined by calculating the saturated current density $\left(J_{0}\right.$, rad $)$ for recombination from the Fourier transform photocurrent spectroscopy (FTPS) spectra fitted by electroluminescence (EL) spectra (Supplementary Fig. 15-18) according to the detailed balance theory 35 . The corresponding non-radiative $V_{\mathrm{oc}}$ losses, $\Delta V_{\mathrm{OC}}$, ${ }_{\mathrm{r}}$, which is the difference between the $V_{\mathrm{OC}}$, rad and the measured $V_{O C}$ from $J-V$ characteristics $\left(V_{O C}\right.$, meas $)$, were determined to be $0.33 \mathrm{~V}, 0.32 \mathrm{~V}$ and $0.26 \mathrm{~V}$ for IDTT-C6-TIC, IDTT-C8-TIC, and IDTT-C10-TIC based OSCs, respectively. 
The energy of charge-transfer $(\mathrm{CT})\left(E_{\mathrm{CT}}\right)$ states were determined by simultaneously fitting EL and FTPS spectra according to Marcus theory ${ }^{36}$ (Supplementary Fig.18) and summarized in Table 2. It is shown that the three systems have different charge transfer states energy of $1.37 \mathrm{eV}, 1.41 \mathrm{eV}$, and $1.50 \mathrm{eV}$ for IDTT-C6-TIC, IDTT-C8-TIC and IDTT-C10-TIC based solar cells, respectively. The differences in $E_{\mathrm{CT}}$ largely explained the variations in $V_{\mathrm{OC}}$ and non-radiative $V_{\mathrm{OC}}$ losses according to energy gap law ${ }^{37}$. The differences in $E_{\mathrm{CT}}$ are most closely related to the LUMO energy differences. The LUMO of IDTT-C10-TIC is $-3.91 \mathrm{eV}$, which is $0.08 \mathrm{eV}$ higher than IDTT-C6-TIC $(-3.99 \mathrm{eV})$ and $0.06 \mathrm{eV}$ higher than that of IDTT-C8-TIC $(-3.97 \mathrm{eV})$. It is notable that this work experimentally proved that the changes in different molecular packing and ordering of NFAs in the BHJ blends significantly affected energy level alignment in the D/A interface and hence the CT state energy as well as the non-radiative $V_{\mathrm{OC}}$ losses of OSCs. Considering the shifting of the charge transfer mode from the conventionally end group p-p stacking (IDTT-C6-TIC) to a mixed transfer mode (IDTT-C8-TIC) and to a p-p stacking free mode (IDTT-C10-TIC), these findings highlight the importance of designing novel molecular semiconductors free from p-pstacking to further reduce the non-radiative recombination losses of OSCs below $0.15 \mathrm{~V}^{38}$.

\section{Fig. 4 | Photovoltaic performance, energy loss analysis and transient absorption kinetics of blend films. a} Current-voltage characteristics of the binary devices based on PBT1-C and IDTT-CX-TIC. $\mathbf{b}$ External quantum efficiencies (EQES) of the binary devices. c,d,e Semi-logarithmic plots of normalized EL (red line), measured EQE (olive line) and EQE calculated by Fourier transform photocurrent spectroscopy (FTPS) (orange dots) as a function of energy for devices based on PBT1-C and different acceptors. Dark gray dot line is black body radiation $\left(\phi_{\mathrm{bb}}\right)$ at $300 \mathrm{~K}$. The ratio of $\mathrm{EL}$ and $\phi_{\mathrm{bb}}$ was used to plot the EQE in the low energy regime (black line). Inset in each figure shows the saturated current density for radiative recombination $\left(J_{0}\right)$, radiative $V_{\mathrm{OC}}$ limit $\left(V_{\mathrm{OC}}\right.$, rad $)$, measured $V_{\mathrm{OC}}\left(V_{\mathrm{OC}}\right.$, meas $)$, and non-radiative $V_{\mathrm{OC}}$ losses $\left(\Delta V_{\mathrm{OC}, \mathrm{nr}}\right) . \mathbf{f}$ Transient absorption kinetics of excitons and charges in the blends, after the selective excitation of the corresponding acceptor phases at $710 \mathrm{~nm}$ at pump fluences of $13 \mu \mathrm{J} / \mathrm{cm}^{2}, 4.7 \mu \mathrm{J} / \mathrm{cm}^{2}$ and $4.7 \mu \mathrm{J} / \mathrm{cm}^{2}$ respectively. The plots are produced via bilinear decomposition of the TA surfaces using spectral mask of excitons from relevant neat acceptor films (Supplementary Fig. 19).

Table 1 | Summary of device parameters and mobility of PBT1-C:IDTT-CX-TIC solar cells

\begin{tabular}{|c|c|c|c|c|c|c|c|}
\hline Active layer & $V_{O C}(V)$ & $\begin{array}{l}J_{s c}(m A \\
\left.\mathrm{cm}^{-2}\right)\end{array}$ & FF (\%) & $\begin{array}{l}\mathrm{PCE}^{\mathrm{a}} \\
(\%)\end{array}$ & $\begin{array}{l}\mathrm{PCE}_{\max } \\
(\%)\end{array}$ & $\begin{array}{l}m_{e}\left(c^{2}\right. \\
\left.v^{-1} s^{-1}\right)\end{array}$ & $\begin{array}{l}m_{h}\left(c^{2}\right. \\
\left.v^{-1} s^{-1}\right)\end{array}$ \\
\hline $\begin{array}{l}\text { PBT1-C:IDTT- } \\
\text { C6-TIC }\end{array}$ & $\begin{array}{l}0.85 \pm \\
0.01\end{array}$ & $17.0 \pm 0.3$ & $\begin{array}{l}66.7 \pm \\
2.4\end{array}$ & $\begin{array}{l}9.6 \pm \\
0.5\end{array}$ & 10.0 & $2.0 \times 10^{-4}$ & $7.3 \times 10^{-4}$ \\
\hline $\begin{array}{l}\text { PBT1-C:IDTT- } \\
\text { C8-TIC }\end{array}$ & $\begin{array}{l}0.88 \pm \\
0.01\end{array}$ & $20.3 \pm 0.2$ & $\begin{array}{l}74.6 \pm \\
1.1\end{array}$ & $\begin{array}{l}13.4 \pm \\
0.2\end{array}$ & 13.7 & $1.2 \times 10^{-4}$ & $6.5 \times 10^{-4}$ \\
\hline $\begin{array}{l}\text { PBT1-C:IDTT- } \\
\text { C10-TIC }\end{array}$ & $\begin{array}{l}0.98 \pm \\
0.01\end{array}$ & $18.1 \pm 0.3$ & $\begin{array}{l}71.3 \pm \\
1.3\end{array}$ & $\begin{array}{l}12.5 \pm \\
0.4\end{array}$ & 12.7 & $6.7 \times 10^{-4}$ & $3.0 \times 10^{-4}$ \\
\hline
\end{tabular}

a The average parameters were calculated from 20 independent cells. 


\section{Table 2 | Measured and calculated parameters to quantify voltage losses}

$\begin{array}{lllllllll}\text { Film } & \begin{array}{l}E_{\mathrm{g}}^{\mathrm{a}} \\ (\mathrm{eV})\end{array} & \begin{array}{l}E_{\mathrm{CT}} \\ (\mathrm{eV})\end{array} & \begin{array}{l}\mathrm{J}_{0, \mathrm{rad}}{ }^{\mathrm{b}}\left(10^{-18} \mathrm{~A}\right. \\ \left.\mathrm{m}^{-2}\right)\end{array} & \begin{array}{l}V_{\mathrm{OC}, \mathrm{sQ}}{ }^{\mathrm{c}} \\ (\mathrm{V})\end{array} & \begin{array}{l}V_{\mathrm{OC}, \mathrm{rad}} \\ (\mathrm{V})\end{array} & \begin{array}{l}V_{\mathrm{OC}, \text { meas }} \\ (\mathrm{V})\end{array} & \begin{array}{l}E_{\text {loss }} \\ (\mathrm{eV})\end{array} & \begin{array}{l}\Delta V_{\mathrm{OC}, \mathrm{nr}}{ }^{\mathrm{d}} \\ (\mathrm{V})\end{array} \\ \begin{array}{l}\text { IDTT-C6- } \\ \text { TIC }\end{array} & 1.60 & 1.37 & 2.5 & 1.32 & 1.18 & 0.85 & 0.75 & 0.33 \\ \begin{array}{l}\text { IDTT-C8- } \\ \text { TIC }\end{array} & 1.59 & 1.41 & 1.6 & 1.31 & 1.20 & 0.88 & 0.71 & 0.32 \\ \begin{array}{l}\text { IDTT- } \\ \text { C10-TIC }\end{array} & 1.61 & 1.50 & 0.27 & 1.33 & 1.24 & 0.98 & 0.63 & 0.26\end{array}$

${ }^{\mathrm{a}} E_{\mathrm{g}}$ was determined from the intersection of the absorption edge and the local EQE maximum. ${ }^{\mathrm{b}} \mathrm{J}_{0, \mathrm{rad}}$ is the saturated current density for radiative recombination, ${ }^{c} V_{\mathrm{OC}, \mathrm{SQ}}$ is the radiative $V_{\mathrm{OC}}$ limit, $V_{\mathrm{OC}, \mathrm{SQ}}$. ${ }^{\mathrm{d}} \Delta V_{\mathrm{OC}, \mathrm{nr}}=V_{\mathrm{OC}, \mathrm{rad}}-V_{\mathrm{OC}, \text { meas }}$

Previous efforts to tune charge transport in organic semiconductors have often come at the expense of exciton transport, prompting us to use femtosecond transient absorption spectroscopy (fs-TAS) to investigate whether these materials suffered this compromise ${ }^{39-41}$. In Fig. $4 \mathrm{f}$, the signals from photogenerated charge populations reach their maximum on the same time-scales ( $50 \mathrm{ps})$ as the complete quenching of the excitons in NFAs. These observations agree with our previous study of NFA blends ${ }^{42-44}$, and imply the NFA excitons can efficiently transport cross to the acceptor phase domain in the BHJ. In the sub-10 ps region, our data analysis shows different prompt charge ratios and charge generation dynamics; however, we are cautious to draw any conclusions from this early time region because charge spectral signatures are buried under very strong exciton signals. We find that shorter exciton lifetimes in the blend compared with the neat NFA films (Supplementary Fig. 20), are consistent with efficient exciton quenching in all cases. Considering the high exciton diffusion coefficients of NFAs, this could still correspond to phase sizes of $10 \mathrm{~s}$ of nanometers ${ }^{45}$. We expect that there may be residual unquenched excitons in the IDTT-C6-TIC blend containing some large phases, however that signature would be overwhelmed by the majority of excitons undergoing distinctly faster charge generation.

Our finding that exciton transport is rather insensitive to molecular packing configurations - unlike charge transport, and contrary to expectations from earlier generations of organic semiconductors reflects the photophysical properties of NFAs. Previous models for exciton transport were dominated by hopping between neighbours, and this coupling for exciton transport is not optimized for the same packing configurations as for charge transport ${ }^{39,40,46}$. On the other hand, NFAs are characterized by strong resonant overlap between their remission and absorption spectra, resulting in self-Förster radius > $4 \mathrm{~nm}^{45}$. Thus, we find that differences in local packing structure do not appear to have a significant effect 
on exciton transport in these materials because of their propensity to undergo through-space exciton hopping over a long distance.

\section{Discussion}

In this work, we demonstrated a simple and effective approach to engineering molecular packing and ordering of NFAs in solid-state BHJ organic photovoltaics. While the recent emergence of advanced NFAs has significantly boosted the PCE of BHJ OSCs by extending the absorption range, improving the charge generation efficiency and minimizing the recombination-related losses, current understanding of charge carrier dynamics and photo-physics is still strongly limited by the empirical model, in which a long-range structural ordering induced by end-group $\pi-\pi$ stacking of NFAs is critical for efficient charge transfer and extraction as well as high photovoltaic efficiency. This statement is particularly true but eliminates the charge transfer/transport channels induced by close atom interactions, as observed in high quality organic crystals based on semiconductors free from $\pi-\pi$ overlap (Herringbone packing) and systematically investigated in this work. The end-group $\pi-\pi$ stacking of NFAs indeed guarantees a high charge carrier mobility and transfer efficiency, however, the ideal long-range structural ordering is difficult to achieve in solid-state thin films, in particular for OSCs based on the BHJ approach. Here we demonstrated that the BHJ thin film free from end-group $\pi-\pi$ stacking (IDTT-C10-TIC) can achieve comparable or even superior photovoltaic properties to the one relies on molecular $\pi-\pi$ stacking induced transport channels. By rationally modifying the length of side-chains, the backbone of NFAs can be manipulated from a strong $\pi-\pi$ stacking mode to an intermixed of $\pi-\pi$ and no $\pi-\pi$ packing mode to refine its solid-state properties.

As it is almost impossible so far to reduce the structural disorder of $\mathrm{BHJ}$ blends, the diverse charge transport channels induced by close side atom interactions and $\pi-\pi$ stacking could be a promising alternative approach enabling sufficient charge transfer efficiency in highly disordered material systems. As a result, the optimized OSCs based on the molecules with intermixed packing mode yielded higher PCEs with high $V_{\text {oc }} s$, and reduced non-radiative recombination losses than those in devices based on molecules with the classic end-group $\pi-\pi$ stacking mode. Therefore, the findings demonstrated in this work not only provide new insights into the effect of NFA molecular packing on exciton dissociation, charge transport, and recombination losses, but also open a new avenue in materials design that endows efficient multiple charge transport channels for next-generation organic photovoltaics.

\section{Methods}

Materials. The polymer donor PBT1-C was synthesized via referencing the reported literature ${ }^{31}$. The detailed synthetic procedures of IDTT-CX-TIC can be found in Supplementary Fig. 21, the corresponding NMR data are included as Supplementary Figs. 22-39 and crystal information of IDTT-CX-TIC is listed in Supplementary Table 3. 
Theoretical Calculation. The details of simulations can be found in Supplementary Note 1. The IDTT-CXTIC crystal based on a supercell $(6 \times 6 \times 4$ for IDTT-C6-TIC; $4 \times 4 \times 3$ for IDTT-C $8-T I C ; 6 \times 3 \times 3$ for IDTT-C10-TIC) was equilibrated at $300 \mathrm{~K}$ for $5 \mathrm{~ns}$ (see Supplementary Fig. 40).

Cyclic voltammetry measurement. Cyclic voltammetry (CV) measurements were performed on a CHI660E electrochemical workstation in a three-electrode cell in anhydrous acetonitrile solvents solution of $\mathrm{Bu}_{4} \mathrm{NPF}_{6}(0.1 \mathrm{M})$ with a scan rate of $50 \mathrm{mV} / \mathrm{s}$ at room temperature under argon. $\mathrm{A} \mathrm{Ag} / \mathrm{Ag}^{+}$wire, two platinum wires were used as the reference electrode, counter electrode, and working electrode, respectively. The materials to be tested in chloroform solution were dried on the surface of the working electrode. The potential of $\mathrm{Ag} / \mathrm{Ag}^{+}$reference electrode was internally calibrated by using ferrocene/ ferrocenium $\left(\mathrm{Fc} / \mathrm{Fc}^{+}\right)$as the redox couple.

Ultraviolet-visible absorption. Ultraviolet-visible absorption spectra were acquired on a UV-vis spectrophotometer (Shimadzu UV-2700). All film samples were spin cast on quartz glass substrates.

TEM. TEM studies were conducted with a JEOL JEM-1400 microscope. The samples for electron microscopy were prepared by dissolving the PEDOT:PSS layer using water and transferring the floating active layer to the TEM grids.

AFM. Atomic force microscopy images were investigated on a Dimension Icon AFM (Bruker) in a tapping mode. All film samples were spin cast on indium tin oxide (ITO)/ZnO substrates.

GIWAXS. The GIWAXS characterization of the thin films is measured on beamline 7.3.3 at the Advanced Light Source (Lawrence Berkeley National Laboratory). All samples are prepared under device conditions on the silicon wafer substrate. The scattering signal of samples is recorded with a pixel size of $0.172 \mathrm{~mm}$ by $0.172 \mathrm{~mm}$ (Pilatus $2 \mathrm{M}$ detector). The distance between the samples and beam center is $\approx 300 \mathrm{~mm}$ which calibrated by the silver behenate standard. The incidence angle is set to be $0.16^{\circ}$. The beam energy is $10 \mathrm{keV}$, operating in top-off mode. A 30 s exposure time on a 2D charge-coupled device (CCD) detector is recorded to collect the diffraction signals. All GIWAXS measurements are done in a helium atmosphere.

RSoXS. The RSoXS is measured at beamline 11.0.1.2 at Advanced Light Source, Lawrence Berkeley National Laboratory. All samples are prepared under device conditions on the Si/PEDOT:PSS substrates. The blend films are then floated in water and transferred to a silicon nitride window. The scattering signals are collected in a vacuum by using a Princeton Instrument PI-MTE CCD (charge-coupled device) camera.

EL and FTPS measurements. Electroluminescence measurements were performed by applying an external voltage/current source through the devices. The luminescence spectra were collected in a backscattering geometry, dispersed by an iHR320 monochromator (Horiba Jobin-Yvon) and recorded with a Peltier-cooled Si CCD (Synapse, Horiba Jobin-Yvon). The FTPS measurements were carried out using a Bruker Vertex 70 Fourier-transform infrared (FTIR) spectrometer, equipped with a quartz tungsten halogen lamp and a quartz beamsplitter as well as an external detector option. A low-noise current amplifier 
(Femto DLPCA-200) was used to amplify the photocurrent produced on illumination of the photovoltaic devices with light modulated by the FTIR. The output voltage of the current amplifier was fed back into the external detector port of the FTIR. Absolute EQE photovoltaic values were redrawn by correcting the FTPS to the EQE of the corresponding solar cells.

Transient absorption measurements. The charge photogeneration and recombination dynamics of polymer: fullerene systems were studied using Femtosecond Transient Absorption (TA) spectroscopy, where the system used is according to the design described in previous literature ${ }^{47,48}$. Here all the polymer:fullerene blends under vacuum were excited by $100 \mathrm{fs}, 580 \mathrm{~nm}$ laser pulses, produced by an optical parametric amplifier (TOPAS) which are chopped at $1.5 \mathrm{KHz}$. The broadband probe pulses are generated by focusing a small fraction of the $800 \mathrm{~nm}$ fundamental into an undoped YAG crystal. The polarization of the pump beam is set at the magic angle (54.70) with respect to that of the probe beam in order to avoid the orientational or polarization effects. After the transmission through the sample, the probe continuum is spectrally dispersed and collected (visible and near IR components) simultaneously by two cameras. The differential transmission signal is calculated from the transmitted probe pulses corresponding to the pump on versus off. A retroreflector on a motorized translational stage is used to vary the relative delay between the pump and the probe pulses. The result of a typical experiment is a 3dimensional data set; where each column corresponds to a full spectrum at a certain time and each raw corresponds to the kinetics at different wavelengths. Exciton dissociation and charge generation dynamics in NFA blends were produced by multivariate curve resolution by alternating least square (MCRALS) method, discussed elsewhere ${ }^{49,50}$. The method includes the bilinear decomposition of the blend TA surfaces by applying spectral masks of excitons from the pristine acceptor films (Supplementary Fig. 19).

Device development and testing. The devices were fabricated with an architecture of ITO/ZnO/active layer $/ \mathrm{MoO}_{3} / \mathrm{Ag}$. The ITO-coated glass substrates were cleaned with sequential ultrasonication in a soapdeionized water mixture, deionized water, acetone and isopropanol. The washed substrates were dried at $110{ }^{\circ} \mathrm{C}$ for one night. The $\mathrm{ZnO}$ precursor solution was spin coated on the ITO substrates at $4000 \mathrm{rpm}$ and the $\mathrm{ZnO}$ layer was generated at $200{ }^{\circ} \mathrm{C}$ for $15 \mathrm{~min}$ in the ambient atmosphere. The substrates were then transferred into a nitrogen-filled glove box. Subsequently, the active layer was spin-coated on the ZnO layer via spin-coating from a chloroform solution of PBT1-C:IDTT-CX-TIC. The $\mathrm{MoO}_{3}(3 \mathrm{~nm})$ and $\mathrm{Ag}$ electrode $(100 \mathrm{~nm})$ were deposited by the sequential thermal evaporation. The current density-voltage $(J-V)$ characteristics of the PV devices were measured under $\mathrm{N}_{2}$ conditions using a Keithley 2400 Source Measure Unit. The currents were measured under $100 \mathrm{~mW} \mathrm{~cm}^{-2}$ simulated $1.5 \mathrm{Global}$ (AM 1.5 G) solar simulator (Enli Technology Co., Ltd, SS-F5-3A). The light intensity was calibrated by a standard Si solar cell (SRC-2020, Enli Technology Co., Ltd). The $J-V$ curves were measured along the forward scan direction from -0.5 to $1.1 \mathrm{~V}$ with a scan step of $50 \mathrm{mV}$ and a dwell time is $10 \mathrm{~ms}$. EQE spectra were carried out using a solar-cell spectral-response measurement system (QE-R, Enlitech). The current density-voltage $(J-V)$ curve of representative devices with various PBT1-C:IDTT-CX-TIC ratio and 
additive volume are shown in Supplementary Figs. 41-46, and the corresponding photovoltaic parameters are listed in Supplementary Tables 4 and 5. We have performed stability analysis (Supplementary Fig. 47)

SCLC mobility measurements. SCLCs were tested in electron-only devices configured with the ITO/ZnO/active layer/ZrAcac/Al and hole-only devices configured with the ITO/PEDOT:PSS/active layer $/ \mathrm{MoO}_{3} / \mathrm{Ag}$. The current density-voltage $(\mathrm{J}-\mathrm{V})$ characteristics of the hole or electron only devices are fitted by the Mott-Gurney law:

$J=(9 / 8) \varepsilon_{r} \varepsilon_{0} \mu\left(V^{2} / L^{3}\right)$

where $J$ is the current density, $\varepsilon_{\mathrm{r}}$ is the dielectric permittivity of the active layer, $\varepsilon_{0}$ is the vacuum permittivity, $L$ is the thickness of the active layer, $\mu$ is the mobility. $V=V_{\mathrm{app}}-V_{\mathrm{b} j}$, where $V_{\mathrm{app}}$ is the applied voltage, $V_{\mathrm{bi}}$ is the offset voltage ( $V_{\mathrm{bi}}$ is $0 \mathrm{~V}$ here). The mobility can be calculated from the slope of the $J^{0.5} \sim V$ curves. Characteristic curves of SCLC devices are shown in Supplementary Fig. 48.

\section{Declarations}

\section{Data availability}

The experiment data that support the findings of this study are available from the corresponding author upon reasonable request.

\section{Acknowledgements}

This work was financially supported by the National Natural Science Foundation of China (NSFC) (Grant Nos. 51825301, 21734001, 51973110, 21734009, 21733005, and 51761135101) and the 111 Project (Grant B14009). F.L. gratefully acknowledges the support by Beijing National Laboratory for Molecular Sciences (BNLMS201902). N.L. gratefully acknowledges the support of 111 project (D18023) and the DFG research grant: BR 4031/13-1. C.J.B. gratefully acknowledges the financial support through the "Aufbruch Bayern" initiative of the state of Bavaria (EnCN and SFF), the Bavarian Initiative "Solar Technologies go Hybrid" (SolTech), and DFG SFB953 (project no. 182849149) and DFG INST 90/917-1 FUGG. Y.S. gratefully acknowledges Prof. Erjun Zhou and Dr. Ailing Tang (NCNST) for the assistance with SCLC measurements. X-ray data were acquired at beam lines 7.3.3 and 11.0.1.2 at the Advanced Light Source, Lawrence Berkeley National Laboratory, which is supported by the Director, Office of Science, Office of Basic Energy Sciences, of the U.S. Department of Energy under Contract No. DEAC02-05CH11231.

\section{Author contributions}

L.Y. synthesized and characterized the IDTT-CX-TIC. K.W. fabricated and characterized the devices. J.X. and F.L. performed the morphology characterization and analysis. X.D., N.L. and C.J.B. studied the energy loss of OSCs. S.C., K.C. and J.M.H. measured TAS and performed the analysis. J.Z., and Z. X. grew IDTT- 
CX-TIC single crystals. S.T. participated in the discussion of IDTT-CX-TIC synthesis. G.H., and Y.Y. calculated the electronic coupling of IDTT-CX-TIC. N.L., F.L. and Y.S. supervised and directed this project. L.Y., K.W., X.D., N.L., F.L. and Y.S. wrote the manuscript. All authors commented on the manuscript.

\section{Competing interests}

The authors declare no competing interests.

\section{References}

1. Cui, Y. et al. Over $16 \%$ efficiency organic photovoltaic cells enabled by a chlorinated acceptor with increased open-circuit voltages. Commun.10, 2515 (2019).

2. Song, J. et al. Ternary Organic Solar Cells with Efficiency $>16.5 \%$ Based on Two Compatible Nonfullerene Acceptors. Mater.31, 1905645 (2019).

3. Jiang, K. et al. Alkyl Chain Tuning of Small Molecule Acceptors for Efficient Organic Solar Cells. Joule 3, 3020-3033 (2019).

4. Meng, L. et al. Organic and solution-processed tandem solar cells with $17.3 \%$ efficiency. Science 361 , 1094-1098 (2018).

5. Lin,Y. et al. 17\% Efficient Organic Solar Cells Based on Liquid Exfoliated WS2 as a Replacement for PEDOT:PSS. Mater.31, 1902965 (2019).

6. Zhan, L. et al. Over $17 \%$ efficiency ternary organic solar cells enabled by two non-fullerene acceptors working in an alloy-like model. Energy Environ. Sci.13, 635-645 (2020).

7. Liu, Q. et al.18\% Efficiency organic solar cells. Bull.65, 272-275 (2020).

8. Cui, Y. et al. Single-Junction Organic Photovoltaic Cells with Approaching 18\% Efficiency. Mater.32, 1908205 (2020).

9. Lin, Y. et al. An electron acceptor challenging fullerenes for efficient polymer solar cells. Mater.27, 1170-1174 (2015).

10. Yuan, J. et al. Single-Junction Organic Solar Cell with over 15\% Efficiency Using Fused-Ring Acceptor with Electron-Deficient Core. Joule3, 1140-1151 (2019).

11. Lin, Y. et al. High-Performance Electron Acceptor with Thienyl Side Chains for Organic Photovoltaics. Am. Chem. Soc.138, 4955-4961 (2016).

12. Zhao, W. et al. Molecular Optimization Enables over 13\% Efficiency in Organic Solar Cells. Am. Chem. Soc.139, 7148-7151 (2017).

13. Zhang, G. et al. Nonfullerene Acceptor Molecules for Bulk Heterojunction Organic Solar Cells. Rev.118, 3447-3507 (2018).

14. Hou, J., Inganas, O., Friend, R. H. \& Gao, F. Organic solar cells based on non-fullerene acceptors. Mater. 17, 119-128 (2018).

15. Yan, C. et al. Non-fullerene acceptors for organic solar cells. Rev. Mater. 3, 18003 (2018). 
16. Baran, D. et al. Reducing the efficiency-stability-cost gap of organic photovoltaics with highly efficient and stable small molecule acceptor ternary solar cells. Mater.16, 363 (2016).

17. Holliday, S. et al. High-efficiency and air-stable P3HT-based polymer solar cells with a new nonfullerene acceptor. Commun.7, 11585 (2016).

18. Ye, L. et al. Quantitative relations between interaction parameter, miscibility and function in organic solar cells. Mater.17, 253-260 (2018).

19. Zhou, Z. et al. High-efficiency small-molecule ternary solar cells with a hierarchical morphology enabled by synergizing fullerene and non-fullerene acceptors. Energy3, 952-959 (2018).

20. Aldrich, T. J. et al. Fluorination Effects on Indacenodithienothiophene Acceptor Packing and Electronic Structure, End-Group Redistribution, and Solar Cell Photovoltaic Response. Am. Chem. Soc.141, 3274-3287 (2019).

21. Han, G., Guo, Y., Song, X., Wang, Y. \& Yi, Y. Terminal $\pi-\pi$ stacking determines three-dimensional molecular packing and isotropic charge transport in an $A-\pi-A$ electron acceptor for non-fullerene organic solar cells. Mater. Chem. C5, 4852-4857 (2017).

22. Shi, X. et al. An Electron Acceptor with Broad Visible-NIR Absorption and Unique Solid State Packing for As-Cast High Performance Binary Organic Solar Cells. Funct. Mater.28, 1802324 (2018).

23. Yao, H. et al. Achieving Highly Efficient Nonfullerene Organic Solar Cells with Improved Intermolecular Interaction and Open-Circuit Voltage. Mater.29, 1700254 (2017).

24. Mai, J. et al. Hidden Structure Ordering Along Backbone of Fused-Ring Electron Acceptors Enhanced by Ternary Bulk Heterojunction. Mater.30, 1802888 (2018).

25. Wang, C., Dong, H., Hu, W., Liu, Y. \& Zhu, D. Semiconducting r-Conjugated Systems in Field-Effect Transistors: A Material Odyssey of Organic Electronics. Rev.112, 2208-2267 (2011).

26. Swick, S. M. et al. Closely packed, low reorganization energy r-extended postfullerene acceptors for efficient polymer solar cells. PNAS115, E8341-E8348 (2018).

27. Li, X. et al. Effects of Short-Axis Alkoxy Substituents on Molecular Self-Assembly and Photovoltaic Performance of Indacenodithiophene-Based Acceptors. Funct. Mater.30, 1906855 (2020).

28. Dai, S. et al. High-Performance Fluorinated Fused-Ring Electron Acceptor with 3D Stacking and Exciton/Charge Transport. Mater.32, 2000645 (2020).

29. Lai, H. et al. Isomer-free: Precise Positioning of Chlorine-Induced Interpenetrating Charge Transfer for Elevated Solar Conversion. iScience17, 302-314 (2019).

30. Fu, H., Wang, Z. \& Sun, Y. Polymer Donors for High-Performance Non-Fullerene Organic Solar Cells. Chem. Int. Ed.58, 4442-4453 (2019).

31. Liu, T. et al. Optimized Fibril Network Morphology by Precise Side-Chain Engineering to Achieve HighPerformance Bulk-Heterojunction Organic Solar Cells. Mater.30, 1707353 (2018).

32. Xia, T., Cai, Y., Fu, H. \& Sun, Y. Optimal bulk-heterojunction morphology enabled by fibril network strategy for high-performance organic solar cells. China Chem.62, 662-668 (2019). 
33. Nikolis, V. C. et al. Reducing Voltage Losses in Cascade Organic Solar Cells while Maintaining High External Quantum Efficiencies. Energy Mater.7, 1700855 (2017).

34. Queisser, W. S. a. H. J. Detailed Balance Limit of Efficiency of p-n Junction Solar Cells. Appl. Phys.32, 510-519 (1961).

35. Rau, U. Reciprocity relation between photovoltaic quantum efficiency and electroluminescent emission of solar cells. Rev. B76, 085303 (2007).

36. Vandewal, K. Interfacial Charge Transfer States in Condensed Phase Systems, Rev. Phys. Chem.67, 113-133 (2016).

37. Benduhn, J. et al. Intrinsic non-radiative voltage losses in fullerene-based organic solar cells. Nat Energy 2, 17053 (2017).

38. Li, N. et al. Analyzing the efficiency, stability and cost potential for fullerene-free organic photovoltaics in one figure of merit. Energy Environ. Sci. 11, 1355 (2018).

39. Menke, M. et al. Tailored exciton diffusion in organic photovoltaic cells for enhanced power conversion efficiency. Nat. Mater. 12, 152-157 (2013).

40. Rim, S.-B., Fink, R. F., Schöneboom, J. C., Erk, P. \& Peumansa, P. Effect of molecular packing on the exciton diffusion length in organic solar cells. Phys. Lett. 91, 173504 (2007).

41. Felter, K. M., Caselli, V. M., Gunbas, D. D., Savenije, T. J. \& Grozema, F. C. Interplay between Charge Carrier Mobility, Exciton Diffffusion, Crystal Packing, and Charge Separation in Perylene DiimideBased Heterojunctions. ACS Appl. Energy Mater.2, 8010-8021(2019).

42. Lin, Y. et al. Mapping Polymer Donors toward High-Efficiency Fullerene Free Organic Solar Cells. Mater.29, 1604155 (2017).

43. Lin, Y. et al. Balanced Partnership between Donor and Acceptor Components in Nonfullerene Organic Solar Cells with $>12 \%$ Efficiency. Mater.30, 1706363 (2018).

44. Dai, S. et al. High-performance organic solar cells based on polymer donor/small molecule donor/nonfullerene acceptor ternary blends. Mater. Chem. A7, 2268-2274 (2019).

45. Chandrabose, S. et al. High Exciton Diffusion Coefficients in Fused Ring Electron Acceptor Films. Am. Chem. Soc.141, 6922-6929 (2019).

46. Mikhnenko, O. V., Blom, P. W. M. \& Nguyen, T.-Q. Exciton diffffusion in organic semiconductors. Energy Environ. Sci. 8, 1867-1888 (2015).

47. Gallaher, J. K. et al. Spectroscopically tracking charge separation in polymer : fullerene blends with a three-phase morphology. Energy Environ. Sci.8, 2713-2724 (2015).

48. Barker, A. J., Chen, K. \& Hodgkiss, J. M. Distance distributions of photogenerated charge pairs in organic photovoltaic cells. Am. Chem. Soc.136, 12018-12026 (2014).

49. Juan, A. d., Jaumot, J. \& Tauler, R. Multivariate Curve Resolution (MCR). Solving the mixture analysis problem. Methods6, 4964-4976 (2014).

50. HOWARD, I. A., MANGOLD, H., ETZOLD, F., GEHRIG, D. \& LAQUAI, F. TRANSIENT ABSORPTION DATA ANALYSIS BY SOFT-MODELLING. Ultrafast Dynamics in Molecules, Nanostructures and Interfaces, 
World Scientific - Series in Optics and Photonics, Vol. 8, World Scientifi c Publishing Co. Pte. Ltd ., Singapore, 53-78 (2014).

\section{Figures}

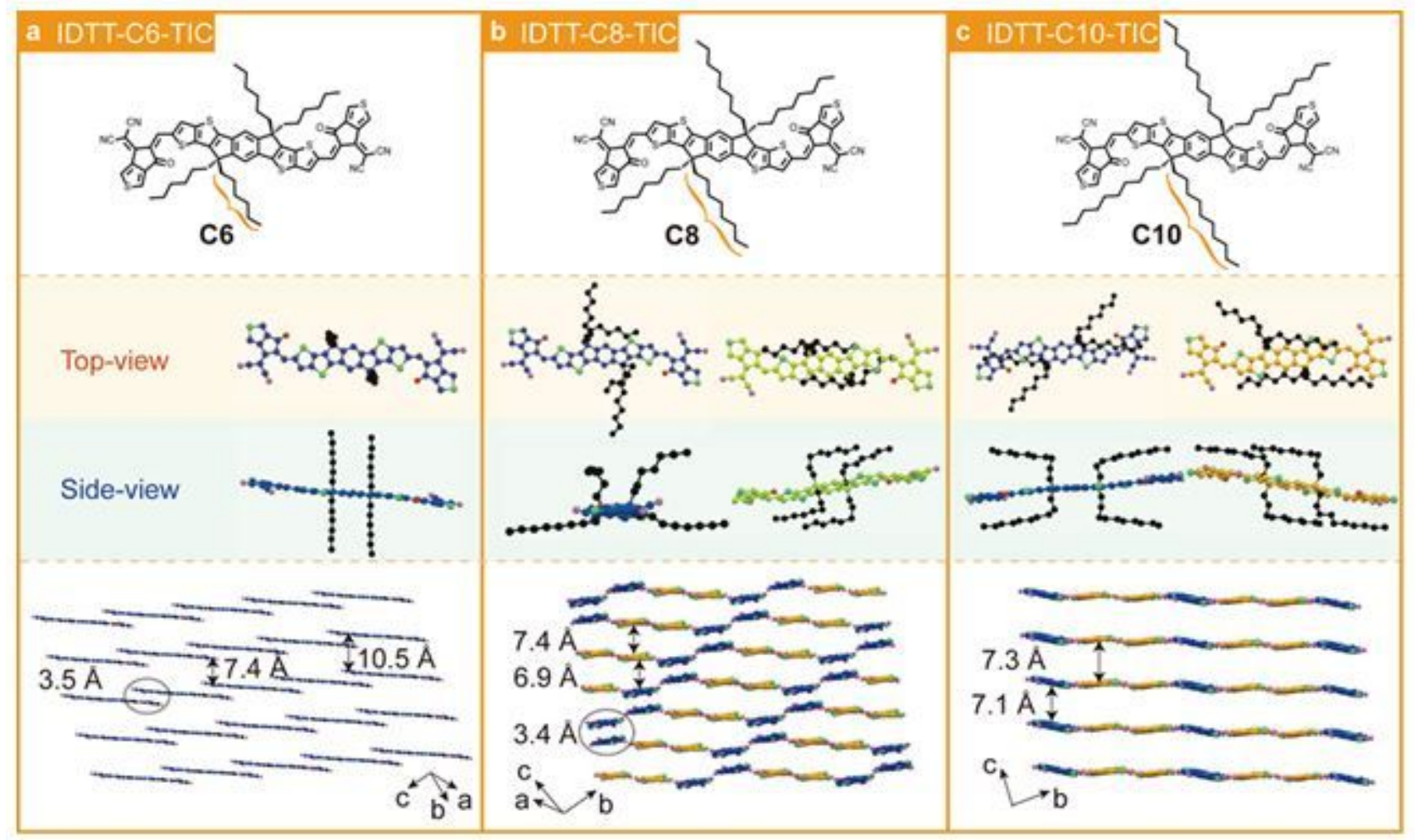

\section{Figure 1}

Molecular and single crystal structure of IDTT-CX-TIC. a The structure of IDTT-C6-TIC. b The structure of IDTT-C8-TIC. c The structure of IDTT-C10-TIC. 

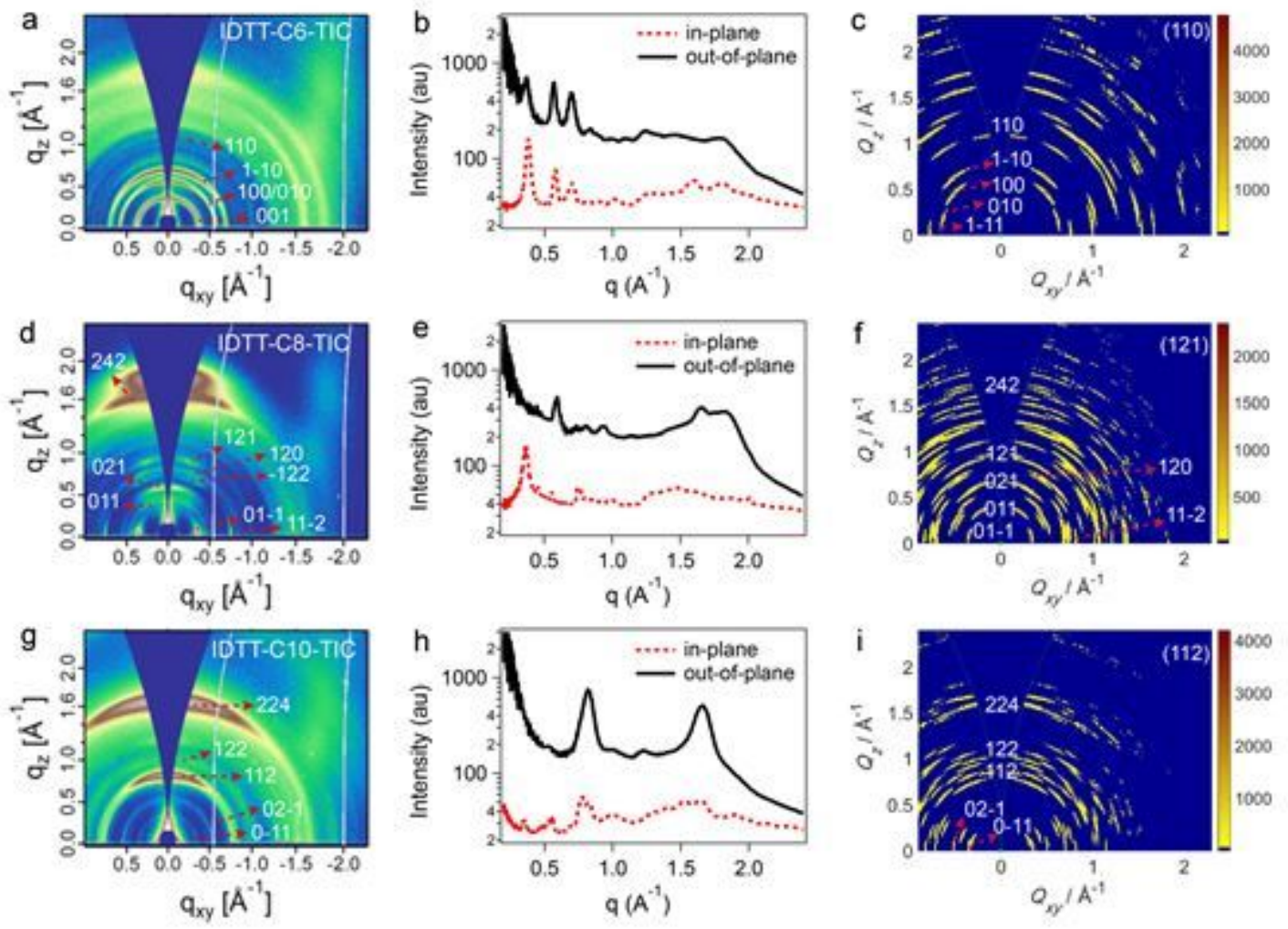

Figure 2

GIWAXS and simulated data. a,d,g GIWAXS diffraction patterns of IDTT-CX-TIC neat films and b,e,h the corresponding in-plane and out-of-plane line-cut profiles. c,f,i The simulated results of IDTT-CX-TIC unit cells.
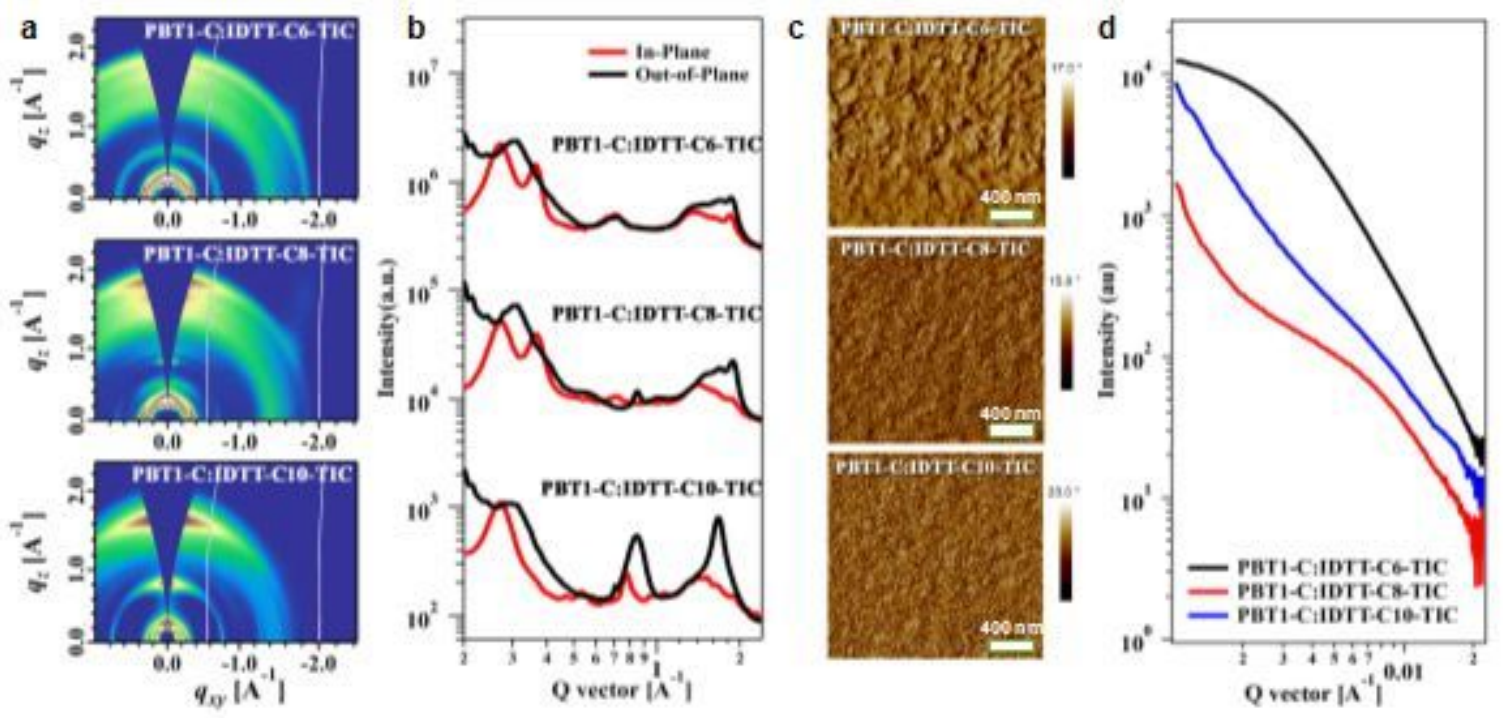

Figure 3

Morphology characterizations. a GIWAXS diffraction patterns of PBT1-C:IDTT-CX-TIC blends and $b$ the corresponding in-plane and out-of-plane line-cut profiles. c AFM phase images of PBT1-C:IDTT-CX-TIC blends $(2 \times 2 \mu \mathrm{m})$. d RSoXS scattering profiles of PBT1-C:IDTT-CX-TIC blends. 

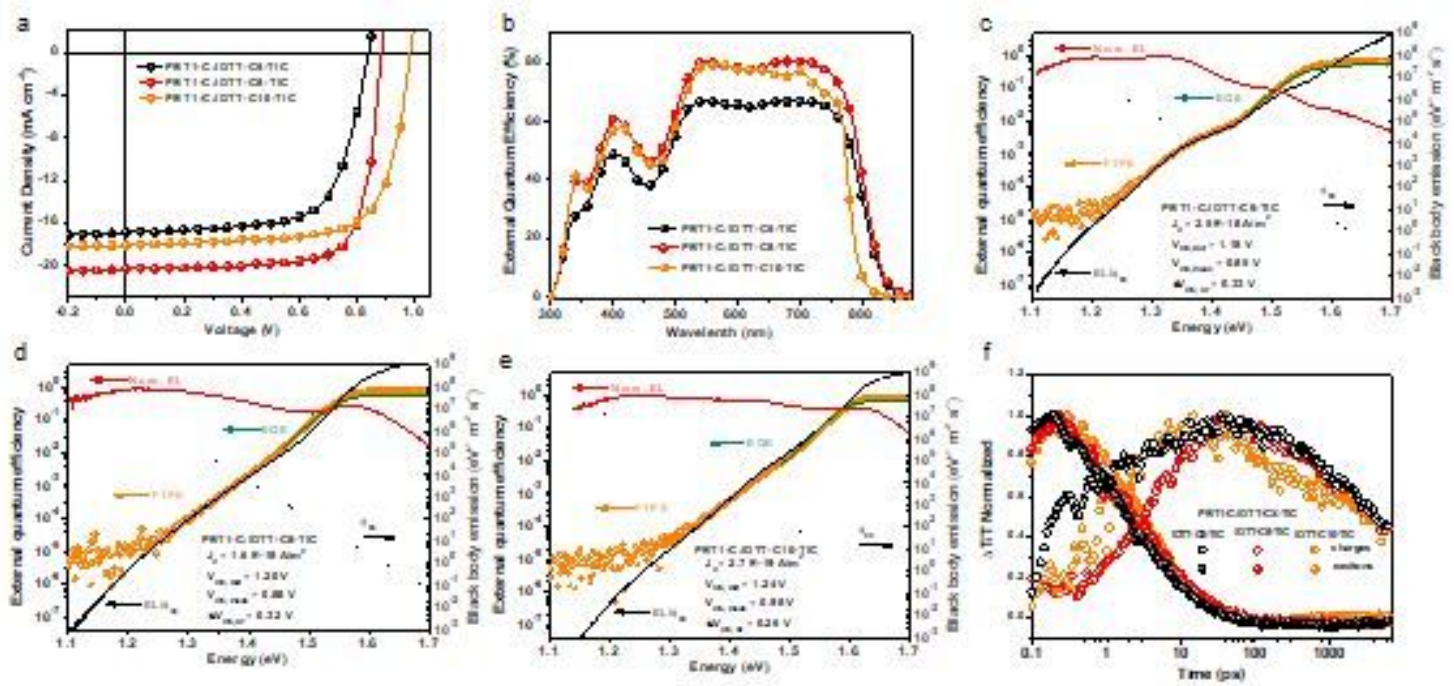

Figure 4

Photovoltaic performance, energy loss analysis and transient absorption kinetics of blend films. a Current-voltage characteristics of the binary devices based on PBT1-C and IDTT-CX-TIC. b External quantum efficiencies (EQEs) of the binary devices. c,d,e Semi-logarithmic plots of normalized EL (red line), measured EQE (olive line) and EQE calculated by Fourier transform photocurrent spectroscopy (FTPS) (orange dots) as a function of energy for devices based on PBT1-C and different acceptors. Dark gray dot line is black body radiation $(\phi \mathrm{bb})$ at $300 \mathrm{~K}$. The ratio of $\mathrm{EL}$ and $\phi \mathrm{bb}$ was used to plot the EQE in the low energy regime (black line). Inset in each figure shows the saturated current density for radiative recombination (JO), radiative VOC limit (VOC, rad), measured VOC (VOC, meas), and non-radiative VOC losses $(\triangle \mathrm{VOC}, \mathrm{nr})$. $\mathrm{f}$ Transient absorption kinetics of excitons and charges in the blends, after the selective excitation of the corresponding acceptor phases at $710 \mathrm{~nm}$ at pump fluences of $13 \mu \mathrm{J} / \mathrm{cm} 2,4.7$ $\mu \mathrm{J} / \mathrm{cm} 2$ and $4.7 \mu \mathrm{J} / \mathrm{cm} 2$ respectively. The plots are produced via bilinear decomposition of the TA surfaces using spectral mask of excitons from relevant neat acceptor films (Supplementary Fig. 19).

\section{Supplementary Files}

This is a list of supplementary files associated with this preprint. Click to download.

- RevisedSupportingInformation.docx

- solar.pdf 